\title{
Curriculum
}

Kardiologe 2021 15:217

https://doi.org/10.1007/s12181-021-00452-1

Angenommen: 18. Januar 2021

Online publiziert: 22. Februar 2021

(c) Deutsche Gesellschaft für Kardiologie -

Herz- und Kreislaufforschung e.V. Published by

Springer Medizin Verlag GmbH, ein Teil von

Springer Nature - all rights reserved 2021

H. Wienbergen ${ }^{1} \cdot$ S. Gielen ${ }^{2} \cdot$ D. Gysan ${ }^{3,6} \cdot$ C. Albus ${ }^{4} \cdot$ U. Landmesser $^{5} \cdot$ R. Hambrecht ${ }^{1}$

'Bremer Institut für Herz- und Kreislaufforschung (BIHKF), Klinikum Links der Weser, Bremen, Deutschland ${ }^{2}$ Klinik für Kardiologie, Angiologie und Internistische Intensivmedizin, Klinikum Lippe, Detmold,

Deutschland

${ }^{3}$ Universität Witten/Herdecke, Witten, Deutschland

${ }^{4}$ Klinik und Poliklinik für Psychosomatik und Psychotherapie, Universitätsklinik Köln, Köln, Deutschland

${ }^{5}$ Medizinische Klinik für Kardiologie, Charité Universitätsmedizin Berlin, Berlin, Deutschland

${ }^{6}$ MVZ Gysan/Heinzler/May (BNK Köln), Köln, Deutschland

\section{Addendum zur Sachkunde „Spezielle kardiovaskuläre Prävention“ der DGK}

\section{Erweiterung Fortbildungsmodul-Nachweise zur Erlangung des Sachkundenachweises}

\author{
Infobox Sachkunde "Spezielle \\ kardiovaskuläre Prävention" der \\ DGK \\ Das Curriculum wurde 2019 in der Zeitschrift \\ Der Kardiologe [1] publiziert.
}

Zum Erwerb der Sachkunde „Spezielle kardiovaskuläre Prävention “ der DGK ist - zusätzlich zu dem Grund- und Aufbaukurs „Spezielle kardiovaskuläre Prävention“" (ggf. zukünftig auch als Online-Kurs) - die erfolgreiche Teilnahme an mindestens einem weiteren Fortbildungsmodul erforderlich.

Zukünftig werden zusätzlich auch folgende Fortbildungsmodule dafür anerkannt:

- Online-CME (Continuing Medical Education) von Präventions-OnlineSeminaren der DGK-Akademie,

- künftige DGK-Akademiekurse oder Online-CME von Online-Seminaren der DGK-Akademie zu präventionsrelevanten Themen wie Atherosklerose und Herz-Diabetes, Psychokardiologie etc.

- Es müssen insgesamt mindestens $6 \mathrm{~h}$ Online-CME zu präventionsrelevanten Themen nachgewiesen sein, damit dies als Fortbildungsmodul für die Sachkunde anerkannt wird.

\section{Korrespondenzadresse}

Prof. Dr. med. H. Wienbergen

Bremer Institut für Herz- und Kreislaufforschung (BIHKF), Klinikum Links der Weser

Senator-Weßling-Str. 1, 28277 Bremen, Deutschland

harm.wienbergen@klinikum-bremen-Idw.de

\section{Prof. Dr. med. R. Hambrecht}

Bremer Institut für Herz- und Kreislaufforschung (BIHKF), Klinikum Links der Weser

Senator-Weßling-Str. 1, 28277 Bremen, Deutschland

rainer.hambrecht@klinikum-bremen-Idw.de

\section{Einhaltung ethischer Richtlinien}

Interessenkonflikt. H. Wienbergen, S. Gielen, D. Gysan, C. Albus, U. Landmesser und R. Hambrecht geben an, dass kein Interessenkonflikt besteht.

Für diesen Beitrag wurden von den Autoren keine Studien an Menschen oder Tieren durchgeführt. Für die aufgeführten Studien gelten die jeweils dort angegebenen ethischen Richtlinien.

\section{Literatur}

1. Wienbergen H, Gielen S, Gysan D et al (2019) Sachkunde „Spezielle kardiovaskuläre Prävention“ der DGK. Kardiologe 13:346-351. https://doi.org/ 10.1007/s12181-019-00347-2 